\title{
Charge Pump DPLL to Operate at High Frequencies
}

\author{
L. Mesquita, T. C. Pimenta \\ Escola Federal de Engenharia de Itajubá \\ Grupo de Microeletrônica \\ Av. BPS 1303, Itajubá - MG. 37500-000 \\ Tel. (+55) 356291190 - 6224450 \\ Fax (+55) 356291187 \\ e-mail: tales@iee.efei.rmg.br
}

\begin{abstract}
This work presents a pre-charge type DPLL that can be used as a clock recovery circuit in SDH/SONET communication protocols at $622 \mathrm{MHz}$. It is presented the phase/frequency detector which can operate equally at low and high frequencies. Each block of the DPLL is described, calculated and simulated. It is also presented the stability criteria and analysis of the DPLL and its final layout. The main feature of this work is the quick synchronization time of the DPLL due to the inclusion of an initialization circuit.
\end{abstract}

Keywords: PLL, DPLL, PFD, VCO, Syncronization.

\section{INTRODUCTION}

In synchronous communication systems, the receiver should be able to recover the clock signal from the incoming message. This is required in order to sample and process the incoming data at the proper time. Therefore, the receiver should be able to have a perfect copy of the clock signal used by the transmitter. The circuit used to recover the clock signal is the clock recover circuit.

The mostly used circuit to recover the clock signal is the Phase Locked Loop PLL [Best,1993; Wolaver,1991]. The PLL compares the phase and/or frequency between the reference signal and a signal generated by the PLL circuit itself. If the reference signal and the self generated signal have the same phase/frequency, they are synchronized, and in this case, the signal generated by the PLL is used as a synchronization signal in the receiver. 
This work presents an implemented Digital Phase Locked Loop - DPLL which has quick synchronization and it is implemented by a reduced number of transistors. This DPLL may be used in Synchronous Digital Hierarchy - SDH and Synchronous Optical Network - SONET network protocols operating at $622 \mathrm{MHz}$.

\section{CHARGE PUMP DPLL}

The DPLL [Paemel,1994] is composed basically of four blocks, namely the Phase Frequency Detector - PFD, the charge pump circuit, the low-pass loop filter and the Voltage Controlled Oscilltor - VCO, as shown in Figure 1.

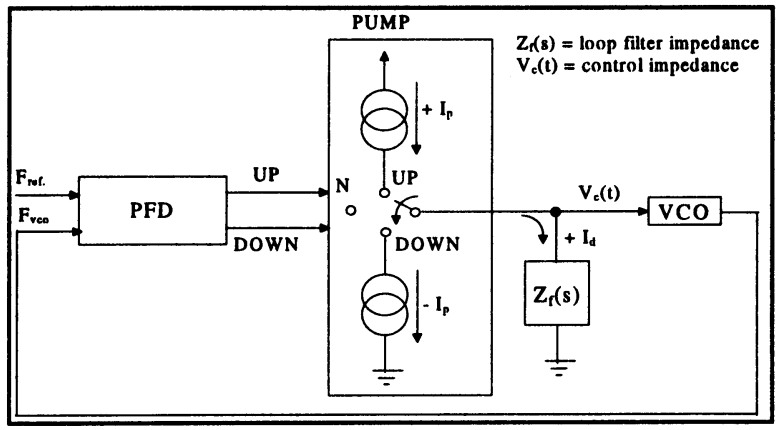

Figure 1 DPLL block diagram.

The PFD is a sequential phase/frequency detector [Gardner,1979]. The PFD has the following valid states as output:

- $\mathrm{Up}=0$ and Down $=0$ indicad high impedance state where $\mathrm{F}_{\mathrm{ref}}=\mathrm{F}_{\mathrm{vco}}$.

- $U p=0$ and Down= 1 indicad Down pulse generated where $F_{\text {ref }}<F_{v c o}$ or $\theta_{\text {ref }}$ leading $\theta_{\mathrm{vco}}$.

- $U p=1$ and Down= 0 indicad Up pulse generated where $F_{\text {ref }}>F_{\text {vco }}$ or $\theta_{\text {ref }}$ lagging $\theta_{\text {vco. }}$.

The PFD needs a charge pump circuit, as indicated in Figure 1. The charge pump is basically an electronic switch that injects (drains) a current $I_{p}$ in(from) the loop filter, depending on the pulse generated by the PFD. The charge pump conducting interval $t_{\text {on }}$ is given by:

$$
t_{o n}=\frac{\left|\theta_{e}\right|}{\omega_{\text {in }}} \text {. }
$$

where $\theta_{\mathrm{e}}$ represents the phase/frequency between the reference and the VCO signals, and $\omega_{i n}$ represents the angular frequency of the incoming signal.

The current injected (drained) by the charge pump circuit, in each cycle, is given by: 
$i_{d}(t)= \pm I_{p}\left(\frac{t_{o n}}{T}\right)= \pm I_{p}\left(\frac{\left|\theta_{e}\right|}{2 \pi}\right)$.

The gain of the charge pump PFD is given by:

$$
K_{d}=\frac{I_{p}}{2 \pi} .
$$

The VCO must produce a signal that has an output frequency as function of the voltage produced by the loop filter and its should have the same phase and frequency of the reference signal.

Since the signal angular frequency is given by the derivative of the signal on the time, them the VCO controlling equation is:

$$
\frac{d \theta_{0}(t)}{d t}=\omega_{0}+K_{0} V_{c}(t)
$$

where $\theta_{0}(t)$ represents the phase of the VCO signal, $\omega_{0}$ represents the VCO angular frequency, and $\mathrm{K}_{0}$ represents the gain of theVCO, given in [rad./V.s].

Therefore, from equation (4), the VCO frequency shift caused by the system controlling voltage is given by (in the frequency domain):

$$
\theta_{0}(s)=K_{0} \frac{V_{c}(s)}{s} .
$$

The signal produced by the PFD is composed of a DC level, proportional to the phase/frequency error of the input signals, and by high frequency components. Since this signal is used as the VCO controlling voltage, the high frequency components must be to eliminated using a low pass loop filter [Gardner,1980; Encinas,1993; Keese,1996]. The loop filter is also responsible to eliminate any ripple in the DPLL, which could take the VCO to saturation, forcing the DPLL to loose the synchronism.

Figure 2 shows the DPLL block diagram. From the figure, the closed and open loop transfer functions are, respectively:

$$
\begin{aligned}
& \frac{\theta_{o}(s)}{\theta_{\text {in }}(s)}=\frac{K_{o} K_{d} Z_{f}(s)}{s+K_{o} K_{d} Z_{f}(s)}, \\
& G_{0}(s)=\frac{K_{d} K_{0} Z_{f}(s)}{s}
\end{aligned}
$$




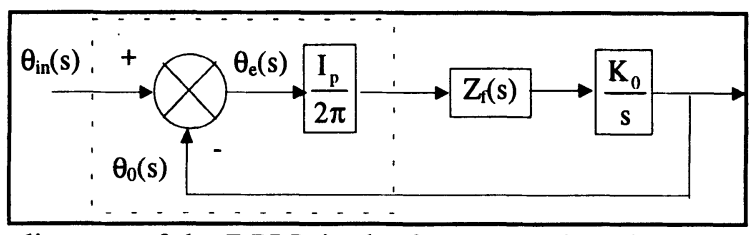

Figure 2 Block diagram of the DPLL in the frequency domain.

\section{PHASE/FREQUENCY DETECTOR}

The implemented PFD operates equally at low and high frequencies [Kodon,1995; Yuan,1989], which does not occur with the conventional PFD [Sayver,1990]. Figure 3 shows the circuit of the PFD which is composed of two blocks reponsible for the generation of the Up and Down signals. Each block is composed of two pre-load branches and a transistor for the RESET of the block. Each block also has an output inverter and a transistor responsible for the PRESET.

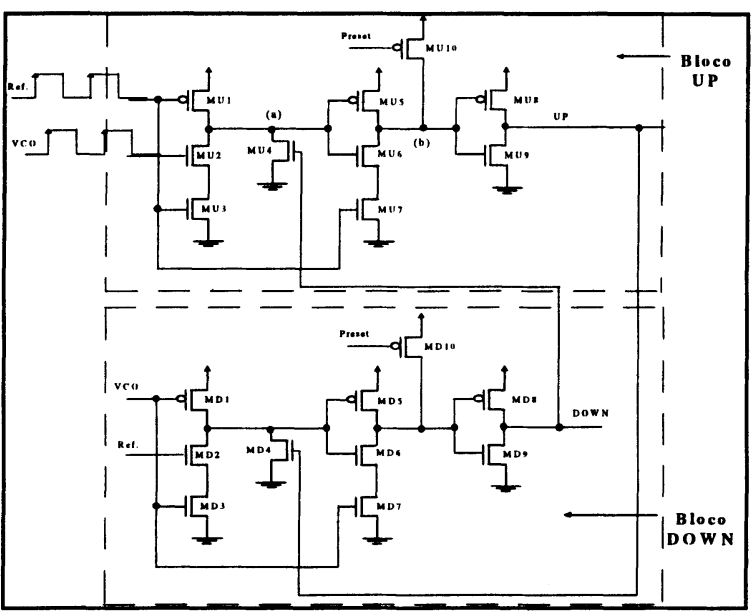

Figure 3 PFD circuit.

The principle of operation of the PFD can be observed considering the generation of an Up signal, as indicated in Figure 4. When the reference signal is low, the precharge transistor $\mathrm{M}_{\mathrm{U} 1}$ conducts, and consequently sets the node (a) to high. Node (b) is also high due to the application of the PRESET signal (system initialization). When the reference signal makes a low-to-high transition, the transistor $\mathbf{M}_{\mathrm{U} 1}$ cuts, which puts node (a) in high impedance. This turns on the transistors $\mathbf{M}_{\mathrm{U} 6}$ and $\mathbf{M}_{\mathrm{U} 7}$, forcing node (b) to low.

At the low-to-high transition of the VCO signal, the transistors $\mathrm{M}_{\mathrm{U} 2}$ and $\mathrm{M}_{\mathrm{U} 3}$ conduct and node (a) goes to low, and consequently the pre-charge transistor $\mathrm{M}_{\mathrm{US}}$ conducts. This situation forces node (b) to high. The signal Up is the inverse of the signal at the node (b). It can be observed from Figure 4 that the Up signal corresponds to the phase difference between the VCO and the reference signals. 


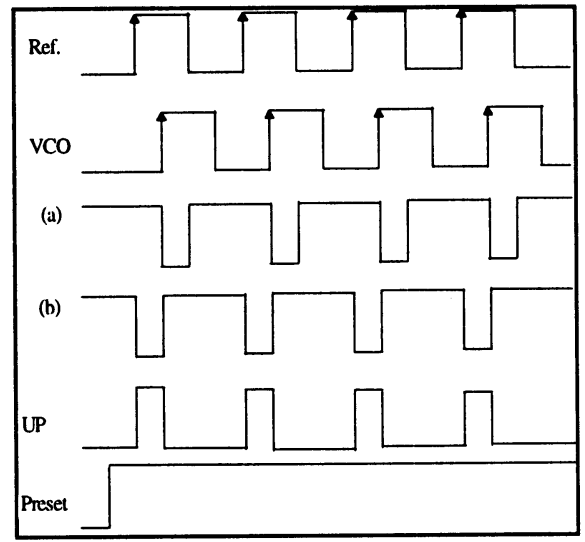

Figure 4 Generation of Up pulse.

Figure 5 shows the HSPICE simulation of this circuit for input frequencies of $100 \mathrm{MHz}, 300 \mathrm{MHz}, 625 \mathrm{MHz}$ and $900 \mathrm{MHz}$.

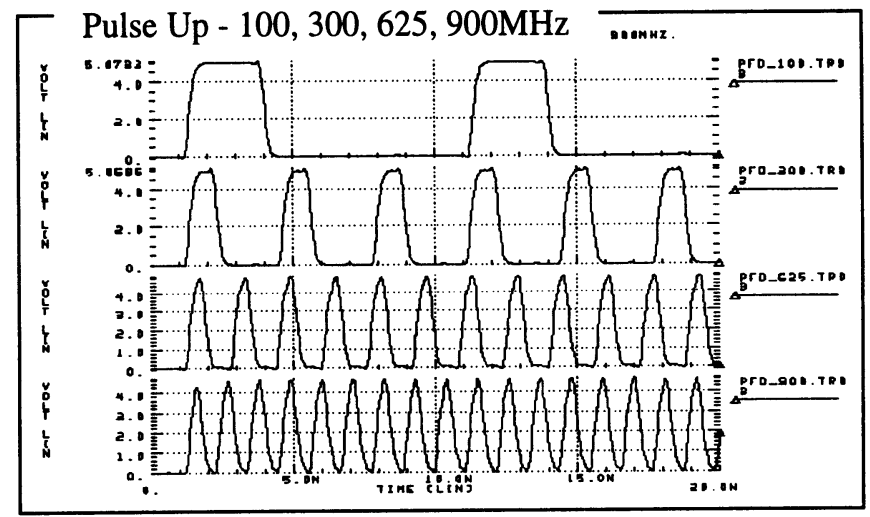

Figure 5 Generation of Up pulses by the PFD.

The charge pump circuit used, shown in Figure 6, is a current switch controlled by the PFD.

Figure 6 Charge pump circuit.

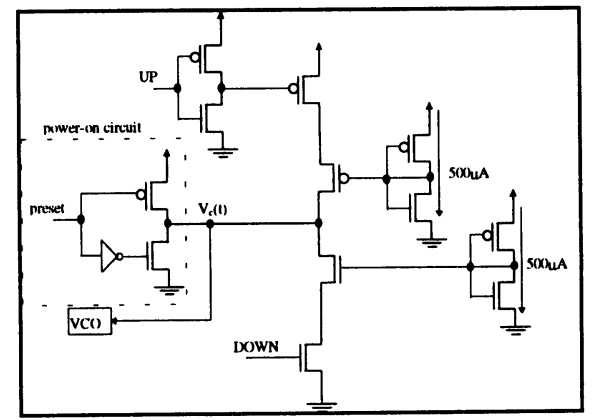


The charge pump has an initialization circuit connected at its output. This circuit places $2.5 \mathrm{~V}$ at the output of the charge pump during the initialization of the DPLL system. Since this signal is applied to the input of the VCO, it starts oscillating at its central oscillating frequency, and consequently the DPLL reduces drastically the synchronization time.

The charge pump sources have been implemented using current mirrors adjusted to $500 \mu \mathrm{A}$. This value of the current has been established to load quickly the internal capacitances and to increase the gain of the DPLL circuit [Keese,1995]. Therefore, the synchronization time is also optimized.

\section{VOLTAGE CONTROLLED OSCILLATOR - VCO}

The voltage controlled oscillator is divided in two blocks, namely the reference circuit and the three stages ring oscillator. The VCO reference circuit is shown in Figure 7.

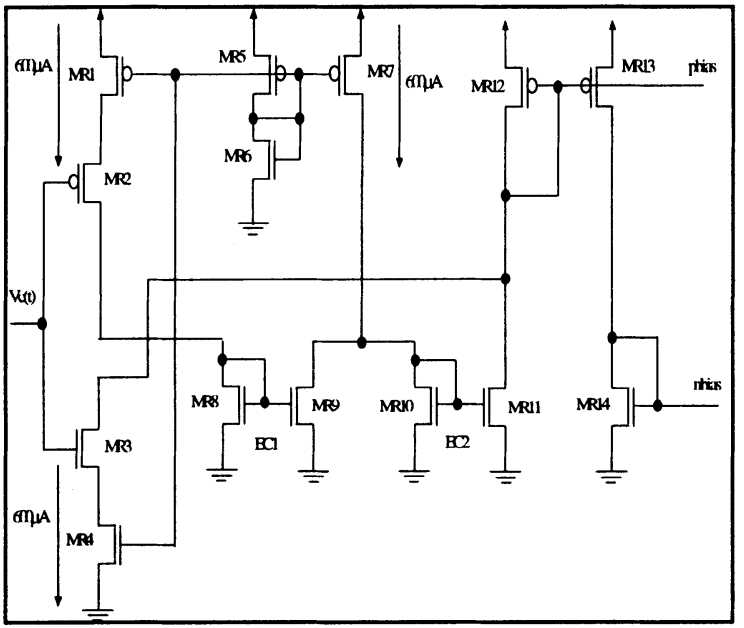

Figure 7 VCO reference circuit

The VCO reference circuit is used to convert the signal from the loop filter in two new signals to be used by the ring oscillator. Considering, initially, that $V_{c}(t)$ is low, them the pMOS input branch (transistors $M_{R 1}$ and $M_{R 2}$ ) conducts a current of $600 \mu \mathrm{A}$. Therefore, the current mirror $\mathrm{EC}_{1}$ starts to conduct, draining all the current $(600 \mu \mathrm{A})$ from the source formed by the transistors $M_{R 5}, M_{R 6}$ and $M_{R 7}$. The output voltage pbias goes to high and nbias goes to low. This situation represents the VCO in its lowest frequency.

In the inverse condition, where the VCO is at the highest frequency, the controlling voltage $\mathrm{V}_{\mathrm{c}}(\mathrm{t})$ is high. This forces the nMOS input branch to conduct, and there will be a maximum current at it output. Therefore, the output voltage pbias goes to low and the nbias goes to high.

Figure 8 shows the controlling voltages of the VCO reference circuit obtained from the HSPICE simulations. 


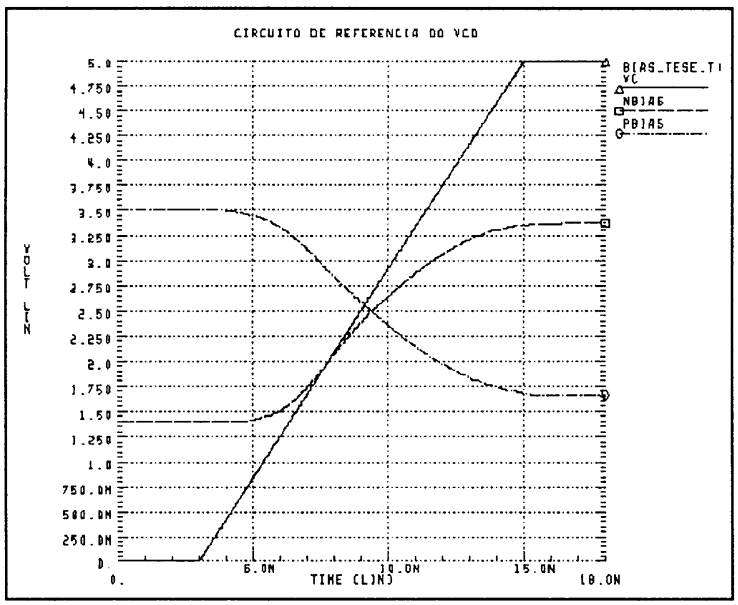

Figure 8 Control voltage of the reference circuit.

The VCO circuit shown in Figure 9 is composed of three stages of current mirrors. The circuit also has a differential amplifier at its output to square the signal generated by oscillator.

The current mirrors $\mathrm{EC}_{1}, \mathrm{EC}_{3}$ and $\mathrm{EC}_{5}$ has a 1:1 current transfer relation, while the current mirrors $\mathrm{EC}_{2}, \mathrm{EC}_{4}$ and $\mathrm{EC}_{6}$ has a 1:2 current transfer relation. The current relation of the mirrors $\mathrm{EC}_{2}, \mathrm{EC}_{4}$ and $\mathrm{EC}_{6}$ has been chosen in such a way that, during operation, they would drain half of the current generated by the transistors $\mathrm{M}_{\mathrm{V} 4}, \mathrm{M}_{\mathrm{V} 9}$ and $\mathrm{M}_{\mathrm{V} 14}$, respectively, but would drain the maximum current from the mirrors $\mathrm{EC}_{1}$, $\mathrm{EC}_{3}$ and $\mathrm{EC}_{5}$.

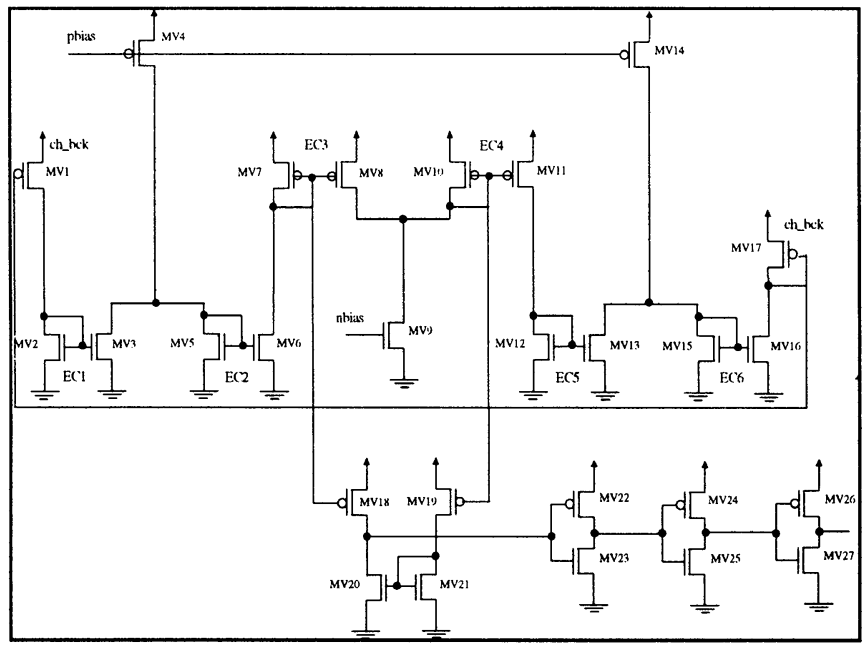

Figure 9 VCO circuit.

When the transistor $\mathrm{M}_{\mathrm{V} 1}$ is conducting (low level at its gate), the current mirror $\mathrm{EC}_{1}$ conducts, thus draining all the current generated by the transistor $\mathrm{M}_{\mathrm{V} 4}$. Therefore, the current mirrors $\mathrm{EC}_{2}$ and $\mathrm{EC}_{3}$ are off. Since the current mirror $\mathrm{EC}_{3}$ is off, the 
current in the transistor $\mathrm{M}_{\mathrm{V} 10}$ (which is part of the current mirror $\mathrm{EC}_{4}$ ) is half of the current produced by the transistor $\mathrm{M}_{\mathrm{vg}}$. The current in $\mathrm{EC}_{4}$ (relation 1:2) and it turns on the current mirror $\mathrm{EC}_{5}$. The current produced by $\mathrm{M}_{\mathrm{V} 14}$ is drained by the current mirror $\mathrm{EC}_{5}$, and the current mirror $\mathrm{EC}_{6}$ cuts off. Therefore, the signal at the gate of the transistor $\mathrm{M}_{\mathrm{V}_{1}}$ goes to high, and the oscillation starts.

The gain of the VCO set by $K_{0}=(\Delta f / \Delta V)$ can be obtained using the VCO characteristic curve shown in Figure 10.

Freq.(MHz)

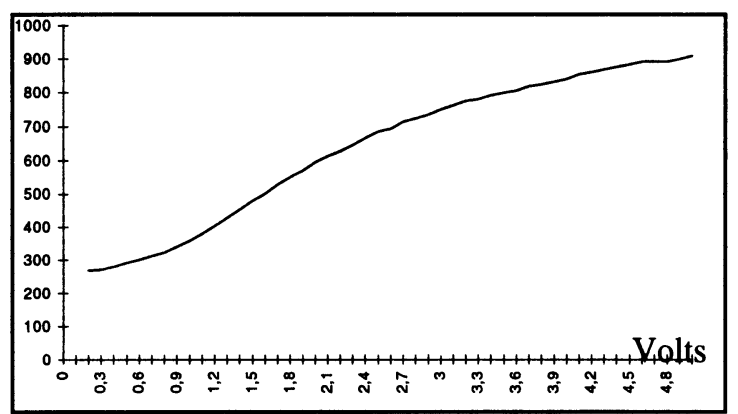

Figure 10 VCO characteristic curve.

\section{LOOP FILTER}

Figure 11 shows the passive second order loop filter designed.

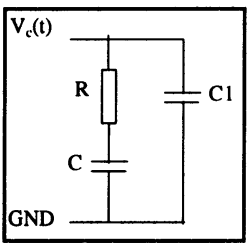

Figure 11 DPLL second order loop filter.

It has been used a second order loop filter to reduce the ripple of the system, as it would occur if a first order loop filter had been used [Gardner,1980; Meyr,1990].

The impedance of the second order loop filter is given by:

$$
Z_{f}(s)=\left(\frac{b-1}{b}\right) \frac{s \tau+1}{s C\left(\frac{s \tau}{b}+1\right)} .
$$

where $b=\left(C / C_{1}\right)+1$, e $\tau=R C$.

The open loop transfer function of the charge pump DPLL using a second order loop filter is: 


$$
G_{0}(s)=K_{0}\left(\frac{I_{p}}{2 \pi}\right) R\left(\frac{b-1}{b}\right) \frac{s \tau+1}{s^{2} \tau\left(\frac{s \tau}{b}+1\right)}
$$

A method of design of DPLL loop filter is to adopt values of cut frequency and phase margin, and them the values of the filter elements can be determined [Keese,1996].

Using Equation (9), the value of the $\tau$ can be determined as a function of $\omega$.

$$
\left.G_{0}(j \omega)\right|_{s=j \omega}=K_{0}\left(\frac{I_{p}}{2 \pi}\right) R\left(\frac{b-1}{b}\right) \frac{j \omega \tau+1}{-\omega^{2} \tau\left(\frac{j \omega \tau}{b}+1\right)} .
$$

The system phase margin, $\mathrm{PM}$ is:

$$
P M=\operatorname{arctag}(\omega \tau)-\operatorname{arctag}\left(\frac{\omega \tau}{b}\right)+180^{\circ} .
$$

The cut frequency can be obtained by taking the derivative of the phase margin by $\omega$ and turning it equal to zero:

$$
\frac{d M F}{d \omega}=\frac{\tau}{1+(\omega \tau)^{2}}-\frac{\left(\frac{\tau}{b}\right)}{1+\left(\frac{\omega \tau}{b}\right)^{2}}=0
$$

$\omega_{c}=\frac{\sqrt{b}}{\tau}$.

H. Meyr and G. Ascheid [Meyr,1990] also deducted an expression to obtain an expression to the DPLL cut frequency as a function of the VCO gain $\mathrm{K}_{0}$, the charge pump output current $I_{P}$, and the value of the DPLL filter resistance $R$, given as:

$$
\omega_{c}=K_{0} \frac{I_{p}}{2 \pi} R\left(\frac{b-1}{b}\right) .
$$

The phase margin of a DPLL system having a second order filter can be adjusted by the proper selection of the value of b. Table 1, obtained using MATLAB, presents a variation phase margin as a function of $b$. 
Table 1 - Variation of Phase Margin as Function of $b$.

$\begin{array}{lllllllll}\text { b } & 6 & 8 & 10 & 12 & 14 & 16 & 18 & 20\end{array}$

$\begin{array}{lllllllll}\mathrm{PM} \text { (Degrees) } & 45 & 51 & 54 & 57 & 60 & 62 & 63,47 & 64,82\end{array}$

Therefore, the design of a loop filter may begin with an adequated value of phase margin, and them Equations (12) and (13) can be used to obtain de values of the filter components.

\section{LAYOUT AND SIMULATIONS}

The final layout of the DPLL circuit, implemented in ES2 $0.7 \mu \mathrm{m}$ CMOS technology, is given in Figure 12. The final simulations, using HSPICE and considering the parasitic capacitances, are shown in Figure 13.

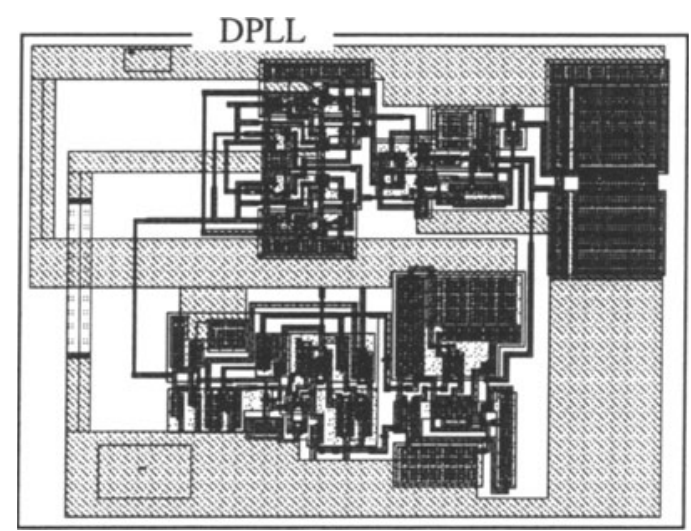

Figure 12 DPLL final Layout

...The DPLL open loop transfer function is given by:

$G_{0}(s)=K_{0}\left(\frac{I_{p}}{2 \pi}\right) R\left(\frac{b-1}{b}\right) \frac{s \tau+1}{s^{2} \tau\left(\frac{s \tau}{b}+1\right)}$.

where $K_{0}=127,8\left(\frac{M H z}{V}\right), I_{p}=500(\mu A), b=11, \tau=R C=1,7.10^{-8}, R=3 K 4(\Omega)$

Then, the DPLL open loop transfer function (Equation 14) becomes:

$G_{0}(s)=\frac{3,357 s+1,9751 \cdot 10^{8}}{2,6273 \cdot 10^{-17} s^{3}+1,70 \cdot 10^{-8} s^{2}}$. 
Figure 14 shows the Bode diagram of this transfer functions.

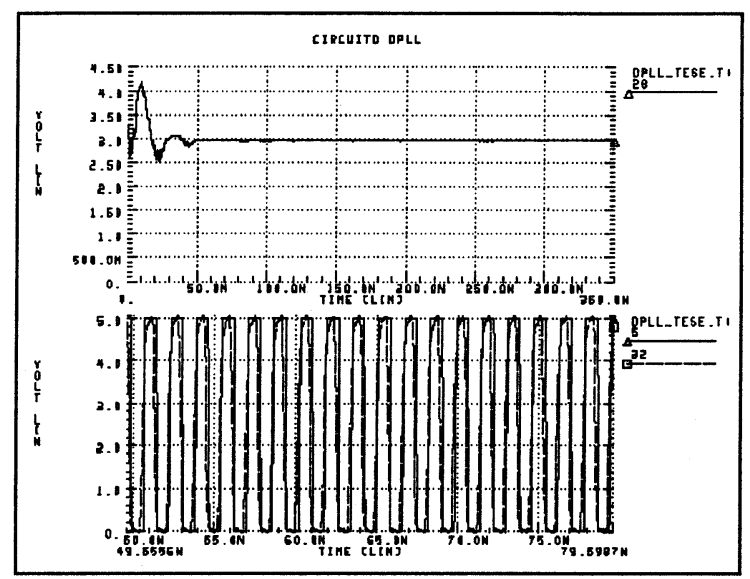

(b)

Figure 13 Simulations of control voltage and synchronized signals.

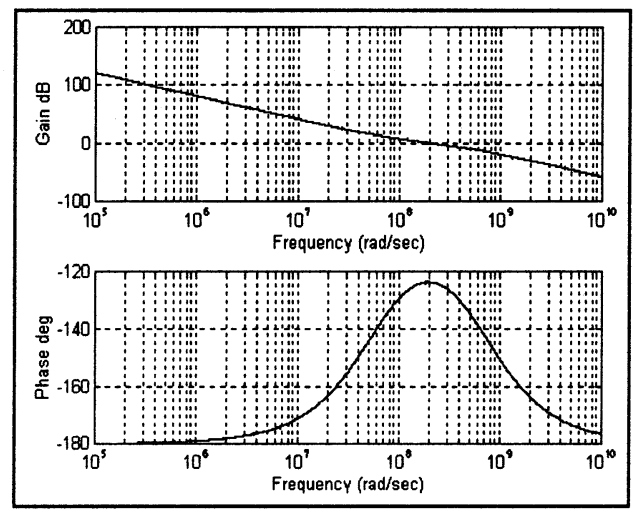

Figure 14 Bode diagram of the DPLL designed.

For the system the phase margin (PM) is:

$$
P M=\arg \left[G_{0}\left(\omega_{c}\right)\right]+180^{\circ}=56,44^{\circ}
$$

where $\omega_{t}$ is DPLL cut frequency.

Since the phase margin is positive, the DPLL is stable, according to the criteria of Nyquist. 


\section{CONCLUSIONS}

This work presented the design of a DPLL that can be used as a clock recover circuit in systems operating at $622 \mathrm{MHz}$ under the SONET/SDH protocols. It has presented the design of each block of the DPLL.

The main feature of the DPLL is its short synchronization time, which was possible due to the use of an initialization circuit in the charge pump and a high gain loop and a loop filter with a short time-constant.

\section{REFERENCES}

Best, R.E. (1993) Phase Locked Loop: Theory, Design and Applications, McGraw Hill.

Encinas, J. (1993) Phase locked loop. Chapmam \& Hall.

Gardner, F.M. (1979) Phaselock Techniques, Jonh Wiley \& Sons.

Gardner, F.M. (1980) Charge pump phase locked loop, IEEE Trans. Comm., vol. $42, n^{\circ} 7$, July, pp. 1849-1858.

Keese, W.O. et. al (1995) A fast locking scheme for PLL frequency synthesizers, National Semiconductor, AN 1000, July.

Keese, W.O. (1996) An analysis and performance evaluation of a passive filter design technique for charge pump phase-locked loops, National Semiconductor, AN 1001, May.

Kondon, H. et. al. (1995) A 1,5V 250Mhz to 3,0V 622Mhz operation CMOS phase locked loop with precharge type phase frequency detector, IEICE Trans Electron, vol. E78C, ${ }^{\circ} 4$, April, pp. 381-388.

Meyr, H.and Ascheid, G. (1990), Synchronization in digital communications. Jonh Wiley \& Sons.

Paemel, M. V. (1994) Analysis of a charge pump PLL: A New Model, IEEE Trans. Comm., vol. 42, n 7,July, pp. 2490-2498.

Sayver, M.and Meyer, R.G. (1990) Frequency limitations of a convencional phase frequency detector, IEEE JSSC, vol. 25, n 4, August, pp. 1019-1022.

Yuan, J. and Svensson, C. (1989) High speed CMOS circuit technique, IEEE JSSC, Vol. 24, n ${ }^{\mathrm{O}} 1$,.February.

Wolaver, D.H. (1991) Phase Locked Loop Circuit Design, Prentice Hall.

\section{BIOGRAPHY}

T. C. Pimenta, $\mathrm{PhD}$ is a Professor, and L.Mesquita, $\mathrm{MsC}$ is a researcher at the microelectronics research and application group of the Federal School of Engineering of Itajubá - EFEI. 